\title{
Investigation of fatigue crack growth under loading sequence effects using in-situ SEM testing
}

\author{
Shan Jiang ${ }^{1}$, Wei Zhang ${ }^{1, *}$, Liang Cai, and Zili Wang $^{1}$ \\ ${ }^{1}$ Science and Technology on Reliability and Environmental Engineering Laboratory, School of Reliability and Systems Engineering, \\ Beihang University, Beijing 100191, China
}

\begin{abstract}
Fatigue damage is one of the most important failure mechanisms in engineering components. The excited structures are usually subjected to spike loads in the fatigue weakness area during their service lives. The nonlinear loading sequence effects due to overloads are significant in the crack propagation process. In this paper, an in-situ scanning electron microscope (SEM) testing is performed to analyse the mechanisms of nonlinear fatigue crack growth affected by the load sequence. The crack tip behaviours under constant amplitude loading cycles superimposed by tensile overload were observed. The SEM experiment results reveal that the overload effects include the transient weakened area (shear bands and micro-cracks) and the relatively long-term retardation. Additionally, the observation loading sequence influence region is larger than the theoretical value. According to these SEM testing analyses, the Willenborg is modified considering the nonlinear loading sequence effects. In this approach, the damage zone concept is introduced to account for the instantaneous acceleration. Moreover, the loading sequence effect area is defined as the whole plastic zone due to overload rather than part of it. The proposed algorithm is validated by experiment data of 350WT steel and Al 2024-T351 specimens under constant loading with overloads. Good agreements are observed.
\end{abstract}

\section{Introduction}

The damage-tolerance design is widely used in many engineering projects. The integrity of damage-tolerance structures subjected to variable amplitude loading during service depends on understanding the fatigue crack growth behavior under such condition. The constant amplitude (CA) loading with overload is a typical scenario in variable loading cases. When the loading spectrum includes multiple overload cycles, the high nonlinearity loading sequence effects have a much stronger influence on fatigue crack growth behavior than under CA loading. Therefore, this investigation focuses on fatigue crack tip behavior and life prediction under constant loading superimposed by overloads.

Many recent monographs present the state-of-the-art in knowledge of fatigue crack growth behavior under overload effects in metallic materials by using experimental investigation and theoretical analysis [1-4]. J. Lankford. JR. and D. L. Davidson examined the fracture surfaces by using the scanning electron microscope (SEM), and further analyzed the plastic zones associated with single overloads [5]. James Lankford and David L. Davidson also performed the SEM overload fatigue tests, in which a special in-situ servo-controlled hydraulic loading stage is used [6]. In their study, the retardation phenomenon was observed directly and the crack tip opening displacements were measured. D. Damri and J. F. K nott observed the fatigue surfaces under the SEM [7]. They aimed to identify the retardation mechanism associated with single overload. These SEM testing observations provide an adequate description of the transient fatigue crack growth behavior under loading sequence effects. Once an overload is applied, the crack growth rate initially increases and then retards in a longer time.

Furthermore, attention is focused on the quantitative prediction of the fatigue life. Wheeler proposed a series of empirical models to account for the overload effects, in which the plastic zone size is a crucial influencing factor of fatigue crack propagation [8]. However, the Wheeler method has several adjustable parameters, which are used to tune the model prediction to fit the actual testing data. And then Willenborg modified these formulations based on physical mechanisms [9]. In this method, the effective stress intensity factor is defined to depict the retardation phenomenon. Many modifications of these two approaches have been proposed in the literatures $[10,11]$. Although most of the existing approaches can quantify the fatigue crack growth retardation after overload, they still offer the most potential improvements. These formulations fall short of calculating the aforementioned initial crack growth acceleration. Therefore, a SEM fatigue testing under CA loading with overload is conducted in this paper. Once an overload is applied, a "damage zone" in the vicinity of the crack tip appears where the potential damage can be seen. After the overload cycle, the crack transient 
acceleration and long-time retardation phenomenon are observed. In the Willenborg model, the overload effects are assumed to be vanished when the current plastic zone reaches the boundary of the previous large plastic zone. Nevertheless, the SEM fatigue tests found that the realistic loading sequence influence area is greater than the theoretical solution. Therefore, based on these SEM experiment results, a modified Willenborg model is proposed. This algorithm can not only explain the retardation phenomenon, but also evaluate the initial acceleration. In this approach, the subsequent loading cycles are under the influence of overload until the crack tip reaches the boundary of the large plastic zone. The testing data of 350WT and aluminum alloy 2024-T351 are employed to validate the proposed approach.

The paper is organized as follows. First, the SEM fatigue testing under CA loading with overload is illustrated. Next, the modified Willenborg model is developed. And then, the prediction results are compared with the fatigue testing data of 350WT and aluminium alloy 2024-T351. Comparison between the proposed approach and the Willenborg model is also performed. Finally, some conclusions are given based on the current investigation.

\section{Experiments}

\subsection{Experiment set-up and procedure}

Crack growth testing were conducted using the edgecrack tension specimens make of Al 2024-T3 in longitudinal (LT) orientation. The configurations of the specimens are: length $=15 \mathrm{~mm}$, width $=10 \mathrm{~mm}$, thickness $=1 \mathrm{~mm}$. The initial crack length is around 1.3 $\mathrm{mm}$. The material components of Al 2024-T3 are listed in Table 1. The mechanical properties are as following: $\sigma_{\mathrm{y}}=345 \mathrm{MPa}, \sigma_{\mathrm{UTS}}=483 \mathrm{MPa}$, Elongation at break $=18 \%$.

Table 1. material component of Al 2024-T3 (Wt.\%)

\begin{tabular}{ccccccccc}
\hline $\mathrm{Al}$ & $\mathrm{Cr}$ & $\mathrm{Cu}$ & $\mathrm{Fe}$ & $\mathrm{Mg}$ & $\mathrm{Mn}$ & $\mathrm{Si}$ & $\mathrm{Ti}$ & $\mathrm{Zn}$ \\
$90.7-94.7$ & $\mathrm{Max} 0.1$ & $3.8-4.9$ & $\operatorname{Max} 0.5$ & $1.2-1.8$ & $0.3-0.9$ & $\operatorname{Max} 0.5$ & $\mathrm{Max} 0.15$ & $\mathrm{Max} 0.25$ \\
\hline
\end{tabular}

A symmetrical loading stage is installed in the Quanta FEG 650 SEM chamber for the high-resolution in-situ observation, as shown in Fig 1. The load capacity of the tensile stage is $2 \mathrm{kN}$. During the testing, the vacuum level is remained to be less than $3 \times 10^{-8} \mathrm{MPa}$.

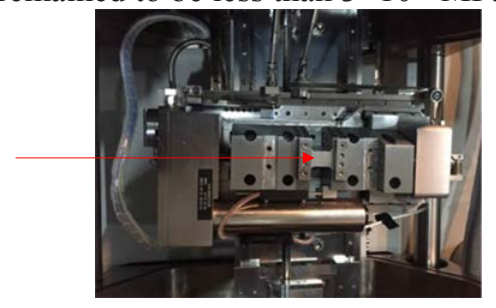

Fig. 1. In-situ SEM fatigue testing setup

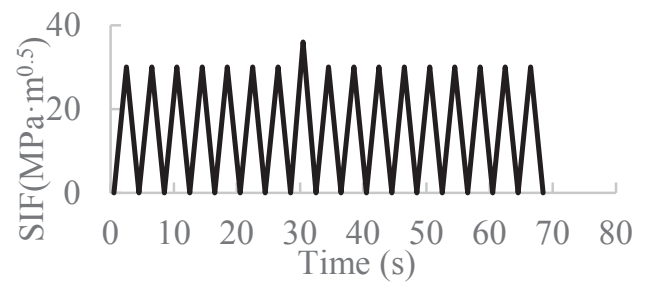

Fig. 2. The loading condition in the SEM fatigue testing

The loading condition is shown in Fig 2. The stress intensity factor (SIF) solution can be expressed as:

$$
\begin{aligned}
& K=Y \cdot \sigma \cdot \sqrt{\pi a} \\
& Y=1.122-0.231 \cdot\left(\frac{a}{b}\right)+10.55 \cdot\left(\frac{a}{b}\right)^{2}-21.71 \cdot\left(\frac{a}{b}\right)^{3}+30.382 \cdot\left(\frac{a}{b}\right)^{4}
\end{aligned}
$$

where $\sigma$ is the applied stress level, $a$ is the crack length, $b$ is the width of the specimen. Firstly, the specimen is subjected to a tension-tension cyclic CA loading sequence with $\mathrm{P}_{\max }=1 \mathrm{kN}, \mathrm{R}=0$. And then an overload is applied. The overload ratio $\mathrm{R}_{\mathrm{OL}}$ is 1.2. Next, continue applying the loads which are same as the previous CA stage. The crack tip behaviour is recorded by the observation system which consists of a metallurgical microscope and a high-resolution imaging acquisition system.

\subsection{Experimental results and discussion}

The SEM fatigue testing results for the specimen subjected to CA loading with overload are shown in this section. In the initial CA loading condition, Fig 3 shows the fatigue surface under peak loading, which demonstrates a smooth and stable crack growth process. The crack plane is approximately flat and perpendicular to the direction of the external applied stress. The average crack growth rate during CA loading is 0.5 $\mu \mathrm{m} /$ cycle, which is a baseline for the following analysis.

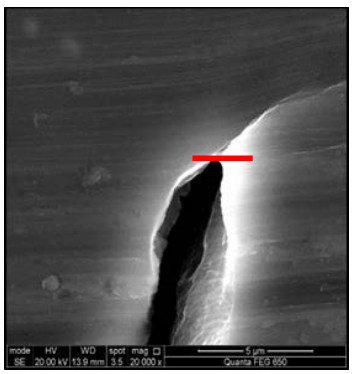

Fig. 3. The crack tip behaviour under peak loading

Fig 4 shows the crack tip behaviour in the overload cycle. A distinct from fatigue surfaces under CA loading, it is apparent that the crack growth zone consisting of potential damage and shear bands are greater. The weaken area in the vicinity of crack is characterized as the damage zone term. This observation implies that instantaneous large load variation enhances the sharply tensile effect to lead to crack growth instability. 


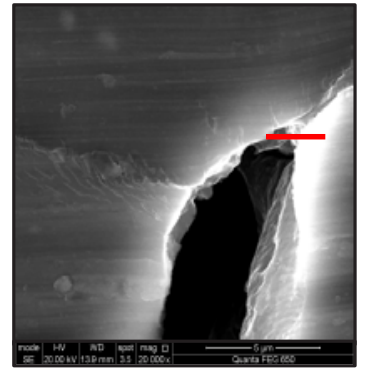

Fig. 4. The crack tip behaviour in the overload cycle

Figs 5.(a)-(c) show the crack tip behaviour during four load cycles after the overload. Under the first peak loading after overload, the crack tip is at point ' $\mathrm{A}$ ' in Fig 5.(a). A micro-crack on the left side of the main crack can be seen, which keeps growing in the next loading cycle (Fig 5.(b)). Ultimately, this micro-crack links up with the principal crack in the fourth cycle, and the crack tip becomes at point ' $\mathrm{C}$ ' in Fig 5.(c), which extremely speed up the crack propagation. The average crack growth rate during these four cycles $(3.04 \mu \mathrm{m} /$ cycle $)$ is much faster than before $(0.5 \mu \mathrm{m} /$ cycle $)$. The appearance of the micro-crack and the linking up process manifest the overload gives rise to the transient crack growth acceleration.

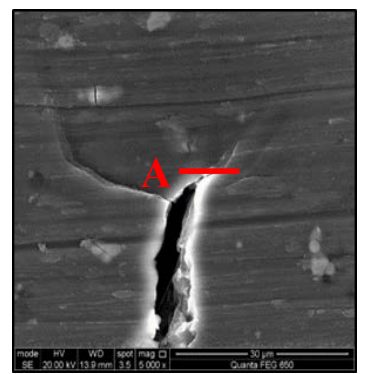

(a) the first peak loading after the overload

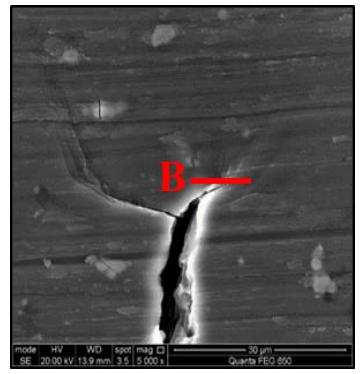

(b) the second peak loading after the overload

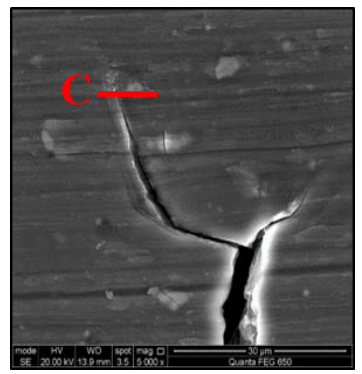

(c) the fourth peak loading after the overload

Fig. 5. The crack tip behaviour right after the overload

Fig 6 shows the crack tip behaviour in the several cycles after the spike load. After 40 cycles, the crack tip reaches point ' $\mathrm{D}$ ' from point ' $\mathrm{C}$ ' in Fig 6. This implies that crack penetrates the aforementioned damage zone and grows slowly.
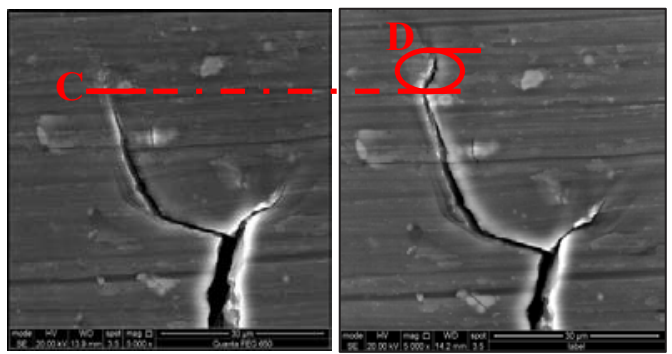

Fig. 6. The crack tip behaviour in several cycles after the overload

The fatigue surface during the crack growth retardation process is magnified, as shown in Fig 7. The magnifying power increases to 20000 from 5000. The crack tip behaviour in 10 cycles are investigated. Although the applied stress levels are the same as the previous CA loading condition, the average crack growth rate during these 10 cycles $(0.27 \mu \mathrm{m} /$ cycle $)$ is awfully smaller than before $(0.5 \mu \mathrm{m} /$ cycle $)$. As a consequence, this SEM fatigue testing reveals that overload will lead to the appearance of damage zone, which elevates the crack growth rate within a transient time. After the acceleration, the crack growth will retard in a relatively longer time.
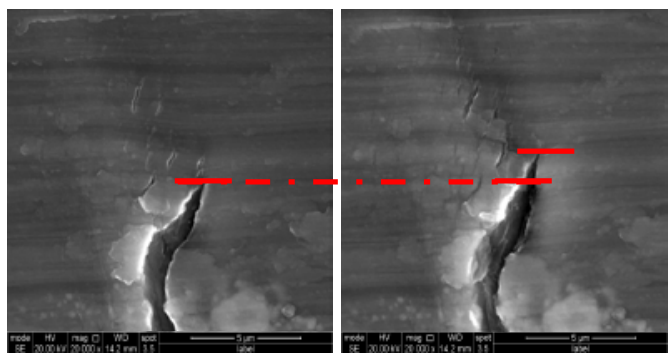

Fig. 7. The crack tip behaviour in 10 cycles during the retardation process

Keeping loading, the crack growth rate eventually returns back the original trend under $\mathrm{CA}$ loading condition. As shown in Fig 8 , the da/dN- $\Delta \mathrm{K}$ data are collected and the Willenborg model is used to calculate the crack growth under CA loading. $\Delta \mathrm{a}$ is the crack increment under the effects of the historical overload, which is around $300 \mu \mathrm{m}$. The corresponding calibration parameters are: $\mathrm{C}=0.0064 \mathrm{e}-6$ and $\mathrm{m}=3.334 . \mathrm{K}_{\mathrm{c}}$ is calculated to be $83 \mathrm{MPa} \cdot \mathrm{m}^{0.5}$. In the Willenborg model, the overload plastic zone is calculated by using the relationship:

$$
Z_{O L . W}=\frac{1}{\eta \pi} \cdot\left[\frac{\left(K_{\max }\right)_{O L}}{\sigma_{y}}\right]^{2}
$$

where the value of $\eta$ is established to be equal to 3 through the analysis [16]. The predicted crack increments under overload effects are $100 \mu \mathrm{m}$, which is much smaller than the experimental value. 


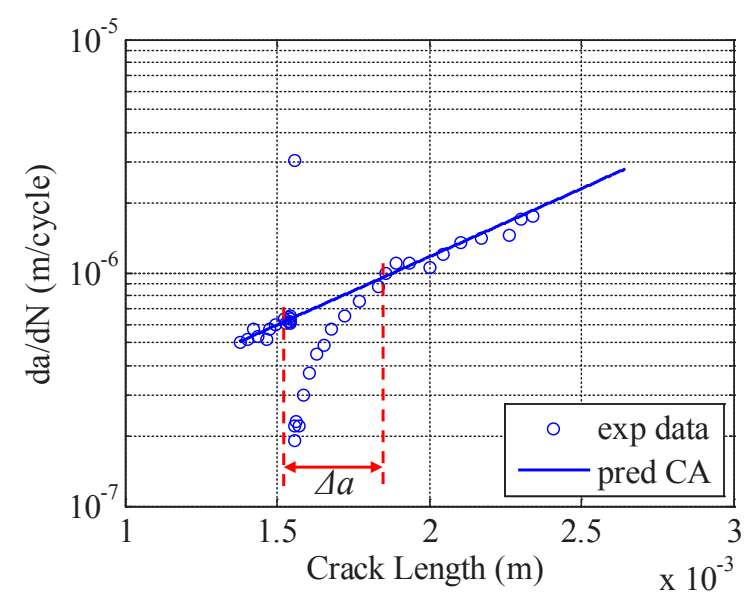

Fig. 8. $a-d a / d N$ curve collected from the SEM fatigue testing

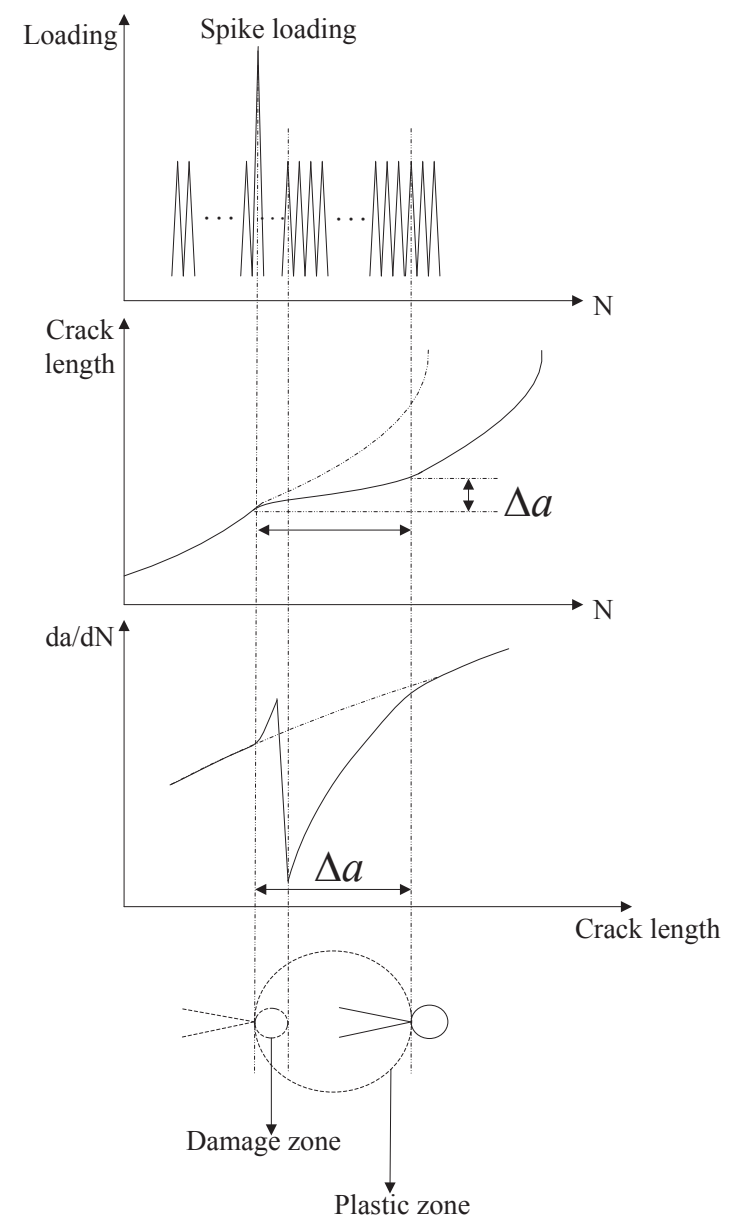

Fig. 9. Schematic illustration of interaction effect

\section{Model development}

According to the SEM fatigue testing observations, the loading sequence effects under CA loading with overloads are investigated, as shown in Fig 9. $\Delta a$ represents the crack increment under the loading sequence effects. Right after the overload, a damage zone appears ahead of the crack tip, in which the crack growth rate instantaneously jumps to a high level. Meanwhile, a large plastic deformation generates, which makes the fatigue crack growth retard in the following cycles. When the crack penetrates the damage zone, the crack growth rate reaches to the minimum value. And then the crack grows through the large plastic zone, the retardation effects gradually relieves and eventually vanishes entirely when the crack tip reaches the boundary of the large plastic zone. The loading sequence effect region is modified to be the whole large plastic zone caused by overload. Afterwards, the crack growth will return back to the original trend.

Willenborg developed a series models based on plasticity physical mechanisms [9]. Current plastic zones are created inside the large plastic zone due to an overload. The retardation phenomenon is described quantitatively by a reduction in stress intensity factor. In this paper, the damage zone concept is used to further modified the Willenborg model, which aims to evaluate the initial acceleration crack propagation process and predict the loading sequence effects more precisely. The effective stress intensity factor can be expressed as:

$$
\begin{gathered}
K_{\text {max.eff }}=K_{\max }-K_{\text {red }} \\
K_{\text {min.eff }}=\left\{\begin{array}{cc}
K_{\min }-K_{\text {red }} & K_{\min }>K_{\text {red }}>0 \\
0 & K_{\min } \leq K_{\text {red }} \leq 0 \text { or } K_{\text {red }} \leq 0<K_{\text {min }}
\end{array}\right.
\end{gathered}
$$

$$
\Delta K_{\text {eff }}=K_{\text {max.eff }}-K_{\text {min.eff }}
$$

where $K_{\text {max.eff }}$ and $K_{\max }$ are the maximum effective and apparent SIF under CA loading, respectively. $\mathrm{K}_{\text {min.eff }}$ and $\mathrm{K}_{\min }$ are the corresponding minimum values; $\Delta \mathrm{K}_{\mathrm{eff}}$ is the effective stress intensity factor range; $K_{\text {red }}$ is the modified stress intensity factor, which characteraizes the loading sequence effects. When $\mathrm{K}_{\text {min }}$ is larger than $\mathrm{K}_{\text {red }}$ and both of these two values are positive, $\Delta \mathrm{K}_{\text {eff }}$ is equal to $\Delta \mathrm{K}$ (the $\mathrm{SIF}$ range). Otherwise, $\Delta \mathrm{K}_{\text {eff }}$ is equal to $\mathrm{K}_{\text {max.eff. }}$ The algorithm of $\mathrm{K}_{\text {red }}$ is modified as:

$K_{\text {red }}=\left\{\begin{array}{cc}{\left[\left(K_{\max }\right)_{O L}-K_{\max }\right] \cdot\left(1-\frac{D_{O L}}{d+\Delta a}\right)^{0.1} \cdot\left(1-\frac{\Delta a}{Z_{O L}}\right)^{0.5}} & d+\Delta a<D_{O L} \\ \left(K_{\max }\right)_{O L} \cdot\left(1-\frac{\Delta a}{Z_{O L}}\right)^{0.5}-K_{\max } & d+\Delta a \geq D_{O L} \text { and } \Delta a \leq Z_{O L} \\ 0 & \Delta a \leq Z_{O L}\end{array}\right.$

where $\left(\mathrm{K}_{\max }\right)_{\mathrm{OL}}$ is the SIF of the overload cycle; $\Delta \mathrm{a}$ is the amount crack increment since the overload cycle; $\mathrm{Z}_{\mathrm{OL}}$ and $\mathrm{D}_{\mathrm{OL}}$ are the plastic zone and damage zone sizes created by overload, respectively; $d$ is the current damage zone. The formulation proposed by Johnson is employed to calculate the plastic zone size, which can be written as [12]:

$$
Z_{O L}=\alpha\left[\frac{\left(K_{\max }\right)_{O L}}{\sigma_{y}}\right]^{2}
$$

where $\sigma_{\mathrm{y}}$ is the tensile yield strength, and $\alpha$ is the plastic zone size factor. Voorwald et al. presented a parametric function for $\alpha$, which is validated by an elastic plastic 
finite element analysis [13, 14]. This equation is given below:

$$
\alpha=\left\{\begin{array}{cc}
\frac{1}{6 \pi} & t \geq 2.5\left(K_{\max } / \sigma_{y}\right)^{2} \\
\frac{1}{\pi} & t \leq \frac{1}{\pi}\left(K_{\max } / \sigma_{y}\right)^{2} \\
\frac{1}{6 \pi}+\frac{5}{6 \pi}\left(\frac{2.5-t\left(K_{\max } / \sigma_{y}\right)^{-2}}{2.5-\pi^{-1}}\right) & \frac{1}{\pi}\left(K_{\max } / \sigma_{y}\right)^{2} \leq t \leq 2.5\left(K_{\max } / \sigma_{y}\right)^{2}
\end{array}\right.
$$

where $t$ is the specimen thickness. The damage zone size is assumed to be proportion to the plastic area size, which can be calculated by the following equation:

$$
D_{O L}=\beta \cdot Z_{O L}=\beta \cdot \alpha\left[\frac{\left(K_{\max }\right)_{O L}}{\sigma_{y}}\right]^{2}
$$

where $\beta$ is the proportionality coefficient. Many experiment results and simulations indicate $\beta=0.05$ is a reasonable evaluation. The Forman equation in conjunction with the Willenborg model can be expressed as [15]:

$$
\begin{gathered}
\frac{d a}{d N}=C \cdot \frac{\left(\Delta K_{e f f}\right)^{m}}{\left(1-R_{e f f}\right) K_{C}-\Delta K_{e f f}} \\
R_{\text {eff }}=\frac{K_{\text {min.eff }}}{K_{\text {max.eff }}}
\end{gathered}
$$

where $\mathrm{C}$ and $\mathrm{m}$ are the material parameters; $\mathrm{R}_{\text {eff }}$ is the effective stress ratio; $K_{C}$ is the critical SIF, which is defined as [15]:

$$
K_{C}=Y \cdot \sigma \cdot \sqrt{\pi a_{c}}
$$

where $\mathrm{Y}$ is the geometry factor of SIF; $a_{c}$ is the critical crack length.

\section{Model validation}

\subsection{Crack growth prediction in 350WT under constant loading with overloads}

An experimental fatigue program was conducted by Taheri et al [16]. The crack growth data on 350WT steel under CA loading with overloads are collected, which are used to validation the proposed model. The rectangular specimens of 300 length $\times 100$ width $\times 5$ thickness $\mathrm{mm}$ is used. The initial centre crack length is $2 \mathrm{a}=20 \mathrm{~mm}$. the mechanical properties of $350 \mathrm{WT}$ in longitudinal (LT) orientation are as follows: $\sigma_{\mathrm{y}}=350$ $\mathrm{MPa}, \sigma_{\text {UTS }}=524 \mathrm{MPa}$, Modulus of elasticity $=205 \mathrm{GPa}$, Poisson's ration $=0.30 . \mathrm{K}_{\mathrm{c}}$ is equal to $110 \mathrm{MPa} \cdot \mathrm{m}^{0.5}$.

Before any prediction can be given, there are still two unknown parameters in Eq.(9). The $d a / d N-\Delta K$ data with $\mathrm{R}=0.3$ in Ref.[17] are used to calibrate these parameters, as shown in Fig 10. The fitting coefficients are $\mathrm{C}=3.793 \mathrm{E}-9$ and $\mathrm{m}=2.365$.

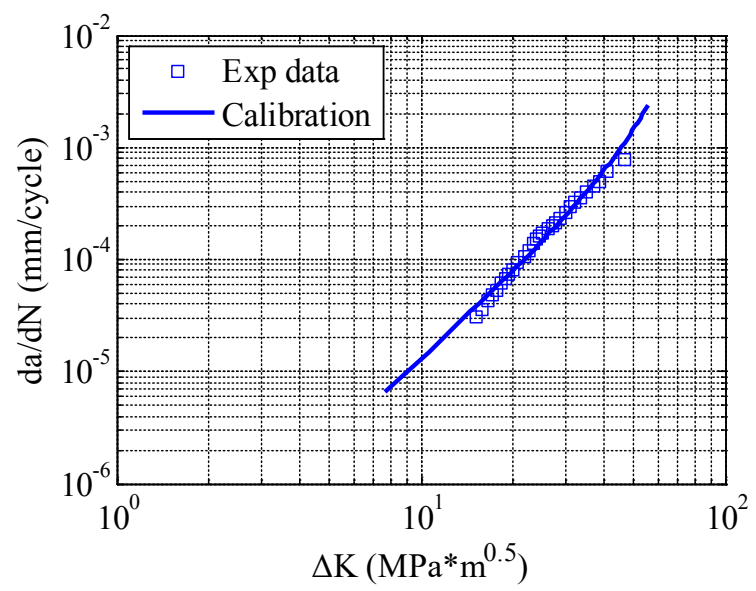

Fig. 10. $d a / d N-\Delta K$ calibration of $350 \mathrm{WT}$

The prediction results are shown in Fig 11. The circles are the testing data. The single overloads are applied at the crack length of 15 and $25 \mathrm{~mm}$. The pink line represents the prediction under CA loading as a baseline. The dashed line is the prediction of Willenborg model, while the full line is the calculation result of modified Willenborg approach. For CA loading condition, there is no different between these two methods. It is clear that the modified approach can give the better predictions under CA loading with overloads.

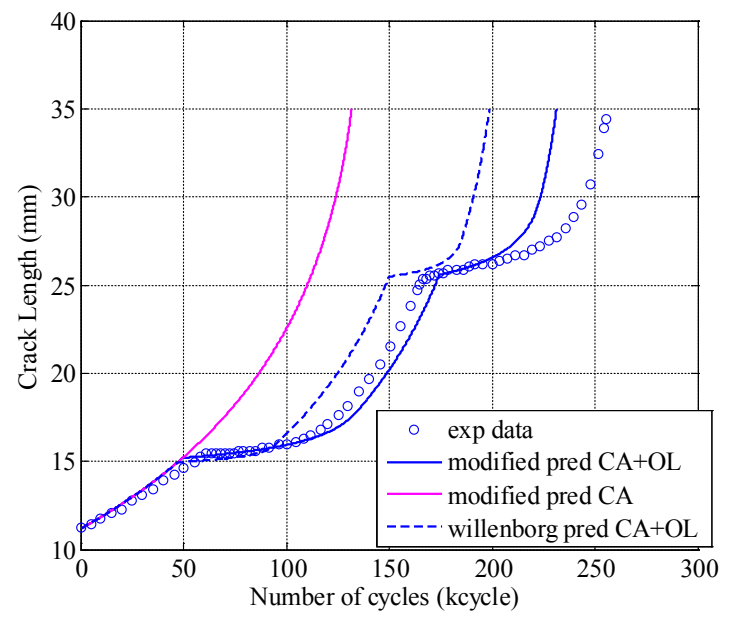

Fig. 11. a-N curves under constant loading with spike load

More detailed information can be obtained in the $\mathrm{da} / \mathrm{dN}-\Delta \mathrm{K}$ curve, as shown in Fig 12 . The Willenborg model falls short of accounting for the initial crack growth acceleration. Additionally, the retardation effects region calculated by Willenborg model is less than testing data. Compared with the Willenborg method, the proposed approach can distinctly match the experimental data better. It is noted that the modified approach is applicable to calculate the fatigue crack growth under the CA loading with overloads. 


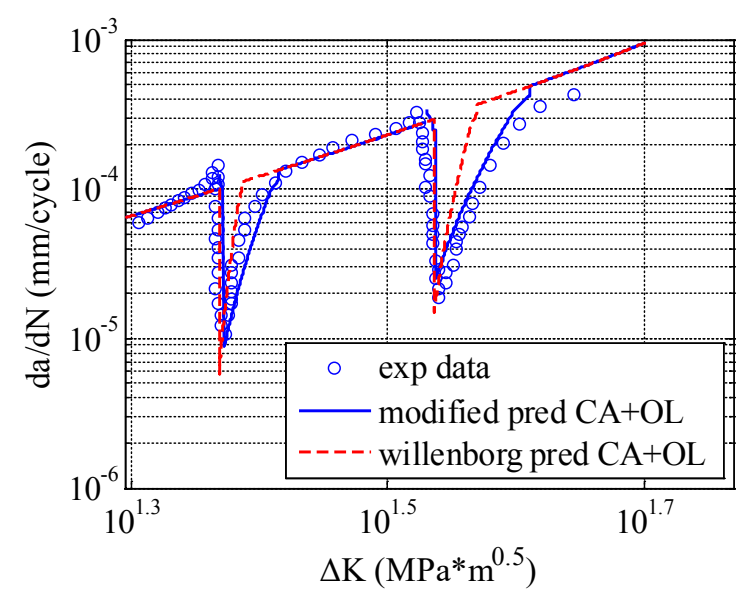

Fig. 12. $\Delta \mathrm{K}-\mathrm{da} / \mathrm{dN}$ curve for constant loading with spike load

\section{2 crack growth prediction in Al 2024-T351 under constant loading with multiple overloads}

The experiment data of 2024-T351 aluminium alloy are also used to validate the presented method [18]. The specimen dimensions are as follows: length $=250 \mathrm{~mm}$, width $=100 \mathrm{~mm}$ and thickness $=6 \mathrm{~mm}$. Initial centre crack length is $2 \mathrm{a}=20 \mathrm{~mm}$. In addition, the mechanical properties of $\mathrm{Al} 2024-\mathrm{T} 351$ in longitudinal (LT) orientation are as follows: $\sigma_{\mathrm{y}}=365 \mathrm{MPa}, \sigma_{\mathrm{UTS}}=466 \mathrm{MPa}$. The value of $\mathrm{K}_{\mathrm{c}}$ is $62.2 \mathrm{MPa} \cdot \mathrm{m}^{0.5}$.

Following the same procedure, the parameters are calibrated by the da/dN- $\Delta \mathrm{K}$ data under CA loading with $\mathrm{R}=0.3$, as shown in Fig 13 [17]. The calibration results are $\mathrm{C}=1.75 \mathrm{E}-9$ and $\mathrm{m}=3.6504$.

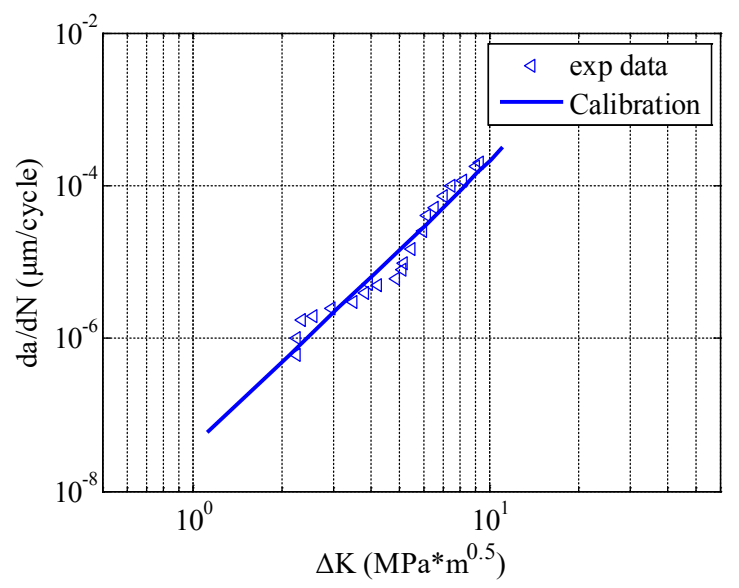

Fig. 13. da/dN- $\Delta \mathrm{K}$ calibration of $\mathrm{Al} 2024-\mathrm{T} 351$

The comparison between the predicted a-N curves and testing data in Al 2024-T351 is shown in Fig 14. The overloads are applied at the crack length of $20 \mathrm{~mm}, 25$ $\mathrm{mm}$ and $32 \mathrm{~mm}$. There are three obvious retarded regions after the overload. These predictions are slightly conservative and follows the same trend as the experiment data.

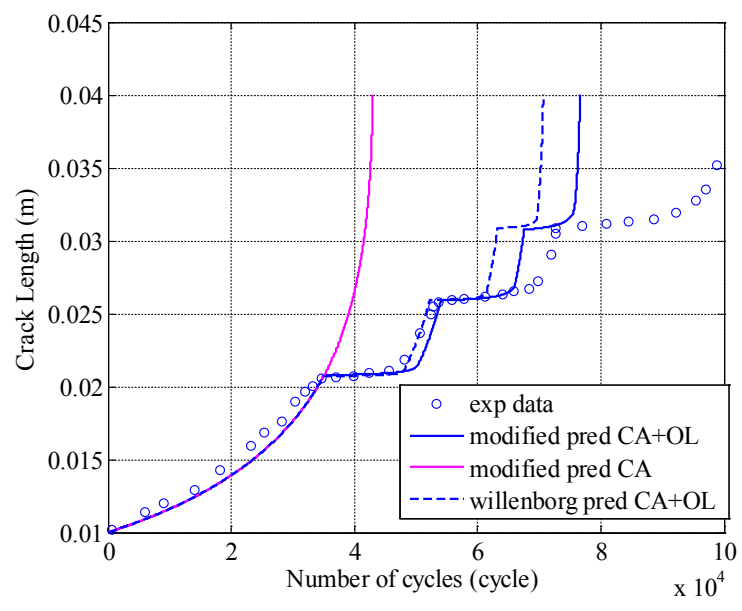

Fig. 14. a-N curves under constant loading with multiple overloads

Similarly, a-da/dN curves are used for further model validation [19], as shown in Fig 15. It is clear that the prediction of the Willenborg model (dashed line) returns back much earlier than the testing data. Therefore, the modified Willenborg method based on the SEM fatigue testing observations can capture the major trends of fatigue life curves under CA loading with overloads.

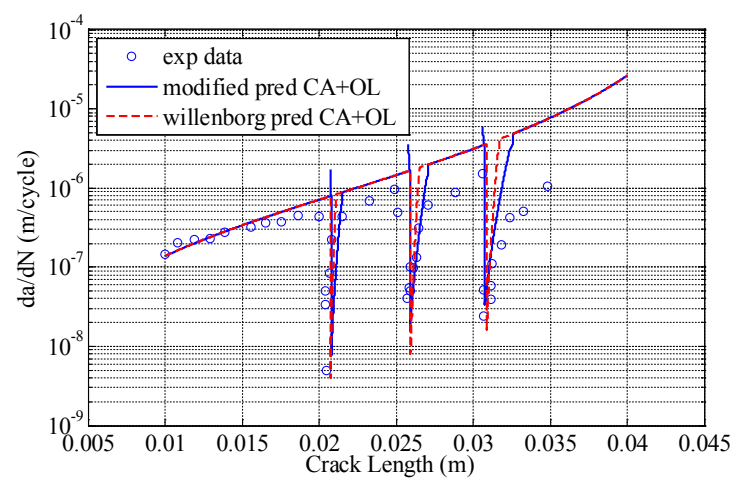

Fig. 15. a-da/dN curve for constant loading with multiple overloads

\section{Conclusions}

An in-situ SEM fatigue testing is conducted to investigate the loading sequence effects caused by overload. Based on these experiment observations, a modified Willenborg model is derived to calculate the fatigue crack growth under CA loading with overloads. This approach is validated by the extensive experiment data of steel and aluminium alloy. The following conclusions can be drawn:

The in-situ SEM fatigue testing under CA loading with overload reveals that a spike load could lead to the transient crack growth acceleration and long-time retardation. Moreover, the observation overload effect area is obviously larger than the theoretical calculation result.

The modified Willenborg approach can not only describe the retardation phenomenon due to overload, but also predict the initial crack growth acceleration. In addition, it manifests that the predicted overload effect 
area matches the testing data better than the Willenborg model by the comparisons. The loading sequence effects could be quantificationally described well by the proposed method.

The research is financially supported by the National Natural Science Foundation of China (Grant No. 51405009).

\section{References}

1. C. Q. Wang, J. J. Xiong, R. A. Shenoi, et al. Int J Fatigue. 83, 280-287 (2016)

2. G. R. Chanani, Effect of thickness on retardation behavior of 7075 and 2024 aluminum alloys, Flaw Growth and Fracture, 365-387 (ASTM International, 1977)

3. T. Zhao, J. Zhang, Y. Jiang, Int J Fatigue. 30(7), 1169-1180 (2008)

4. S. Y. Lee, P. K. Liaw, H. Choo, et al. Acta Mater. 59(2), 485-494 (2011)

5. J. Lankford, D. L. Davidson, J Eng Mater-T ASME. 98(1), 17-23 (1976)

6. J. Lankford, D. L. Davidson, The effect of overloads upon fatigue crack tip opening displacement and crack tip opening closing loads in aluminium alloys. ICF5, Cannes (France) (1981)

7. D. Damri, J. F. Knott, Fatigue Fract Eng M. 14(7), 709-719 (1991)

8. O. E. Wheeler, J Basic Eng-T ASME. 94(1), 181186 (1972)

9. J. Willenborg, R. M. Engle, H. A. Wood. A crack growth retardation model using an effective stress concept. No. AFFDL-TM-71-1-FBR, Air Force Dynamics Lab Wright-Patterson Afb On, (1971)

10. B. K. C. Yuen, F. Taheri. Int J Fatigue. 28(12), 1803-1819 (2006)

11. J. P. Gallagher, A generalized development of yield zone models. No. AFFDL-TM-FBR-74-28, AIR FORCE FLIGHT DYNAMICS LAB WRIGHTPATTERSON AFB OH, (1974)

12. W. S. Johnson, Multi-parameter yield zone model for predicting spectrum crack growth. Methods and Models for Predicting Fatigue Crack Growth Under Random Loading. ASTM STP 748, J. B. Chang and C. M. Hudson, Eds., American Society for Testing and Materials, 85-102 (ASTM International, 1981)

13. H. J. C. Voorwald, M. A. S. Torres, C. C. E. P. Júnior. Int J Fatigue. 13(5), 423-427 (1991)

14. X. Huang, M. Torgeir, W. Cui. Int J Fatigue. 30(1), 2-10 (2008)

15. R. G. Forman, V. E. Kearney, R. M. Engle. J Basic Eng-T ASME. 89(3), 459-463 (1967)

16. F. Taheri, D. Trask, N. Pegg. Mar Struct. 16(1), 6991 (2003)

17. X. Huang, M. Torgeir, W. Cui. Int J Fatigue. 30(1), 2-10 (2008)
18. F.J. McMaster, D. J. Smith. Int J Fatigue. 23, 93-101 (2001)

19. J. T. Liu, P. A. Du. Zhang Z Y. Sci China Technol Sc. 55(3), 673-683 (2012) 